\title{
Finite mass effects in Higgs production in association with jets
}

\author{
Nicolas Greiner \\ Physik-Institut, Universität Zürich, Wintherturerstrasse 190, CH-8057 Zürich, Switzerland \\ E-mail: greiner@physik.uzh.ch
}

\section{Stefan Höche}

SLAC National Accelerator Laboratory, Menlo Park, CA 94025, USA E-mail:

shoechedslac.stanford.edu

\section{Gionata Luisoni*}

Theoretical Physics Department, CERN, Geneva, Switzerland

E-mail: gionata.luisonidcern.ch

\section{Marek Schönherr}

Physik-Institut, Universität Zürich, Wintherturerstrasse 190, CH-8057 Zürich, Switzerland

E-mail: marek.schoenherr@physik.uzh.ch

\section{Jan-Christopher Winter}

Department of Physics and Astronomy, Michigan State University, East Lansing, MI 48824, USA

E-mail: jwinter@pa.msu.edu

\begin{abstract}
The production of a Higgs boson at the LHC suffers from large higher order corrections. This is true also when it is accompanied by further jets. The most precise results rely on computations in an effective theory where the heavy quark loops, mediating the coupling between the Higgs boson and the gluons, are integrated out. As the LHC is delivering more and more precise data, it is important to understand in detail the validity range of such effective theory predictions, in particular in view of boosted analyses. In this talk we will present detailed comparisons between effective theory results and predictions obtained in the full theory.
\end{abstract}

XXV International Workshop on Deep-Inelastic Scattering and Related Subjects 3-7 April 2017

University of Birmingham, $U K$

\footnotetext{
*Speaker.
} 


\section{Introduction}

The production of a Higgs boson in gluon-gluon fusion is the leading Higgs production channel. Its production in association with jets is also very important, in particular for the determination of differential spectra. However, being a loop-induced process, increasing the multiplicity or the precision of the predictions becomes quickly very hard or beyond the possibilities of current computational techniques. When the mass of the fermions running in the loop is much larger than the Higgs boson mass, the heavy fermion can be integrated out and the coupling between gluons and the Higgs can be described by an effective vertex, simplifying the calculations considerably. The validity of this effective theory is however known to be limited, in particular when the momentum flow through the effective vertex becomes of the same order as the fermion masses. In this talk, based on the results presented in Ref. [1], we explore the range of validity and the breakdown of the effective theory approach at a more quantitative level.

\section{Computational setup}

In the following we will compare predictions for the production of a Higgs boson in association with up to three jets at Leading Order (LO) in the full Standard Model (SM) with Next-toLeading Order (NLO) predictions in the effective theory presented in Refs. [2, 3]. All the one-loop amplitudes are computed with GoSAM[4], a publicly available package for the automated generation of one-loop amplitudes. For the generation of the amplitudes GoSAM employs QGRAF [5], FORM [6] and SPINNEY [7] whereas for the reduction it relies on NINJA [8], which implements an algorithm based on the Lauren expansion [9], SAMURAI [10], which uses the OPP reduction method [11] in $d$ dimensions, or GoLEM95C [12], which is a tensor integral library. For the latter the reconstruction of the tensor structure is performed either using the method outlined in [13] or via derivation with respect to the loop momentum. The scalar loop integrals can be computed with QCDLOOP [14] or ONELOOP [15]. All the tree-level parts are computed with the multipurpose Monte Carlo (MC) program SHERPA [16], using Comix [17], to which the 1-loop code is interfaced via the BLHA interface [18]. LO and NLO events are stored in form of RоOT Ntuples [19]. For more details about the calculation, the Ntuples format and the values of the physical parameters we refer to the original publication [1].

\section{Results}

We present results for $\mathrm{H}+1,2$ and 3 jets using the following set of baseline cuts at a center-ofmass energy of $13 \mathrm{TeV}$ :

$$
p_{T, \text { jet }}>30 \mathrm{GeV}, \quad\left|y_{\text {jet }}\right|<4.4
$$

where jets are clustered with the anti- $k_{T}$ algorithm [20] as implemented in the FASTJET package [21] with radial distance $R=0.4$. For the $100 \mathrm{TeV}$ results we increase the transverse momentum cut to $p_{T, \text { jet }}>100 \mathrm{GeV}$. The factorization and renormalization scales are set equal to

$$
\mu_{F}=\mu_{R} \equiv \frac{\hat{H}_{T}^{\prime}}{2}=\frac{1}{2}\left(\sqrt{m_{H}^{2}+p_{T, H}^{2}}+\sum_{i}\left|p_{T, i}\right|\right) .
$$



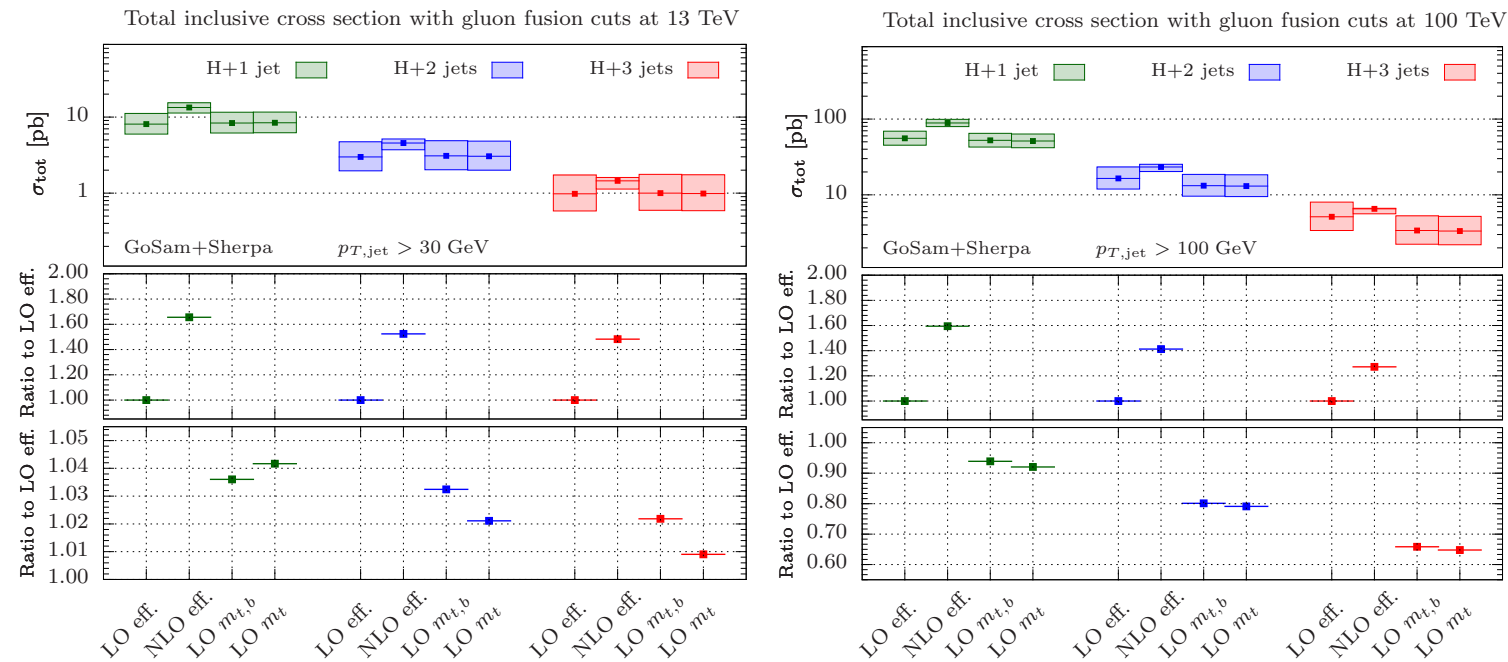

Figure 1: Inclusive cross sections for $\mathrm{H}+1$ jet, $\mathrm{H}+2$ jets and $\mathrm{H}+3$ jets production at center-of-mass energies of $13 \mathrm{TeV}$ and $100 \mathrm{TeV}$ shown to the left and right, respectively. The width of the bands shows the associated scale uncertainty.

We start comparing the predictions for the total cross section for 13 and $100 \mathrm{TeV}$ shown in Fig. 1. The height of the boxes represents the uncertainty due to renormalization and factorization scales variation by factors of 0.5 and 2 . For each multiplicity we show the prediction at LO and NLO in the effective theory (labeled as $\sigma_{\mathrm{LO}, \text { eff }}$ and $\sigma_{\mathrm{NLO}}$, eff respectively) and compare them with the full theory results at leading order when considering both top-quark and bottom-quark loops, called $\sigma_{\mathrm{LO}, m_{t, b}}$, as well as with the case where only top-quark loops are taken into account, labeled $\sigma_{\mathrm{LO}, m_{t}}$. In the lower plots we include the ratios to the leading order results in the effective theory. Focusing on the central values, we observe that the leading-order contribution in the effective theory agrees in general very well with the predictions based on the full theory. Taking bottom-quark loops into account leads to corrections, which are as small as one percent for all three final-state multiplicities we are considering, and, as expected, they become even smaller at $100 \mathrm{TeV}$. However, it is interesting to note the change in the sign of these corrections with increasing jet multiplicity. While for $\mathrm{H}+$ 1 jet production at $13 \mathrm{TeV}$ the cross section is reduced when bottom-quark loop contributions are included, for $\mathrm{H}+2$ jets and $\mathrm{H}+3$ jets the cross section increases instead. This is due to a destructive interference effect on the cross section in the low transverse momentum region caused by terms which scale like

$$
\frac{1}{p_{T}^{2}} \frac{m_{b}^{2}}{m_{H}^{2}} \log ^{2}\left(\frac{m_{b}^{2}}{p_{T}^{2}}\right)
$$

which for $m_{b}<p_{T}<m_{H}$ can become important in $\mathrm{H}+1$ jet. For higher multiplicities the transverse momentum $p_{T}$ is diluted among more jets, leading to a ratio in the logarithm closer to unity. Therefore $\sigma_{\mathrm{LO}, m_{t}}<\sigma_{\mathrm{LO}, m_{t, b}}$ for $\mathrm{H}+2$ jets and $\mathrm{H}+3$ jets. At $100 \mathrm{TeV}$ the minimum jet transverse momentum is much harder, and the phase space region where terms of the form of Eq. 3.3 could become important are excluded. For this reason the hierarchy between $\sigma_{\mathrm{LO}, m_{t}}$ and $\sigma_{\mathrm{LO}, m_{t, b}}$ is the same in this case for $\mathrm{H}+1,2$ and 3 jets.

We now turn our attention to the observables displaying the breakdown of the effective theory prediction par excellence, which are the transverse momentum of the Higgs boson, $p_{T, H}$, and 

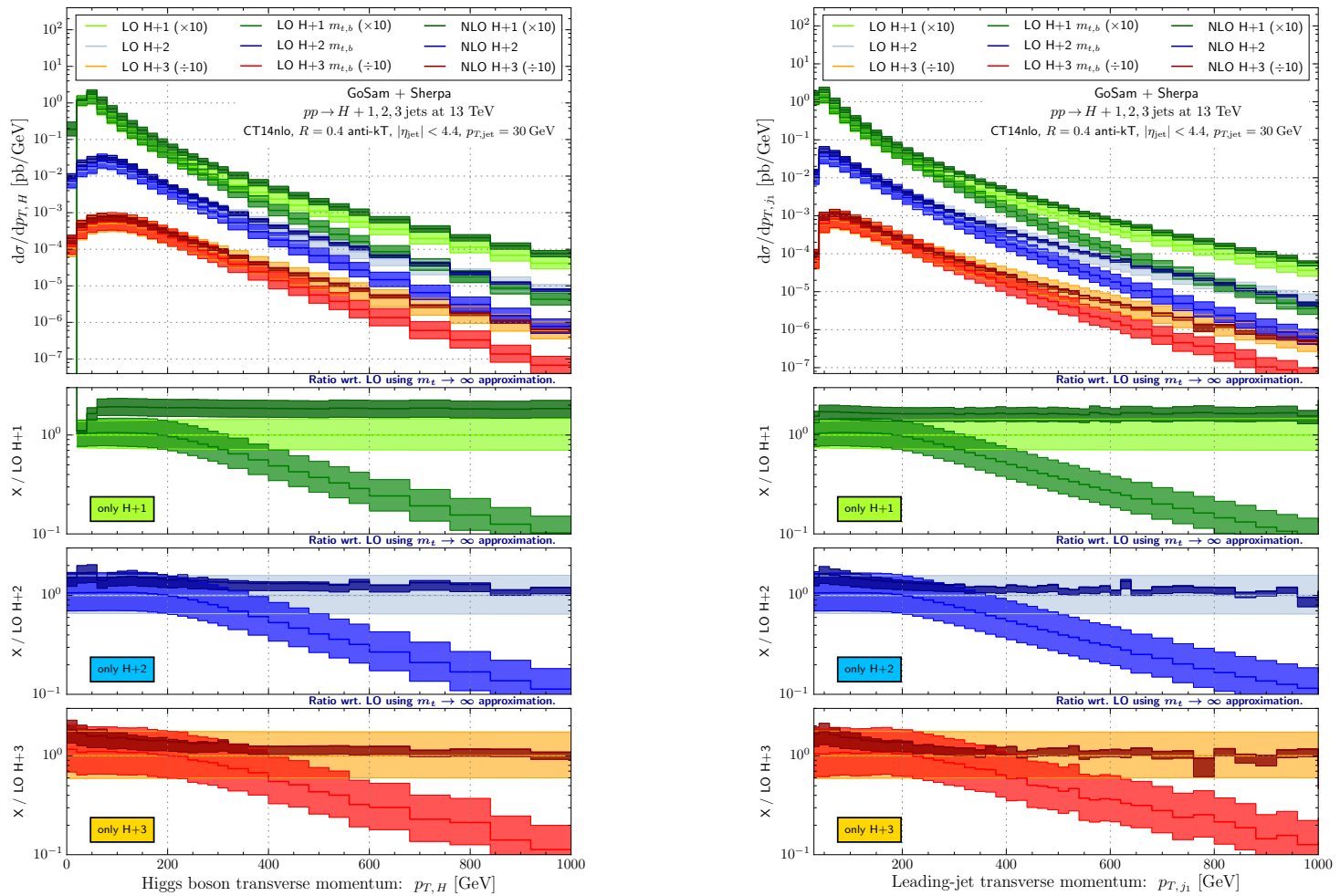

Figure 2: Comparison of effective theory predictions at LO and NLO with LO predictions (indicated by the extra ' $m_{t, b}$ ' label) obtained in the full SM for the Higgs boson transverse momentum $p_{T, H}$ (left) and the leading jet transverse momentum, $p_{T, j_{1}}$ (right). Note that the $\mathrm{H}+1$ jet (green curves) and $\mathrm{H}+3$ jets (red curves) predictions have been rescaled for better visibility. The smaller plots in the lower part of each panel show the ratios of the three different predictions normalized to the LO effective theory prediction. This is done separately for each of the $\mathrm{H}+1$ jet, $\mathrm{H}+2$ jets and $\mathrm{H}+3$ jets processes.

the one of the leading jet, $p_{T, j_{1}}$, shown to the left and right in Fig. 2, respectively. These two distributions clearly show the expected behaviour of $p_{T}$-tail softening. In order to better quantify the scaling properties of the distributions at large transverse momenta we introduce the quantity $R_{m_{t, b}}(O)$ which is defined as

$$
R_{m_{t, b}}(O) \equiv \frac{\left.\frac{\mathrm{d} \sigma}{\mathrm{d} O}\right|_{m_{t, b}}}{\left.\frac{\mathrm{d} \sigma}{\mathrm{d} O}\right|_{\mathrm{eff} .}} .
$$

We observe that the point at which the effective theory approach starts to break down occurs around Higgs boson or lead-jet values of $p_{T}=200 \mathrm{GeV}$ and is to a good approximation independent of the jet multiplicity of the Higgs boson production processes. Above this scale, the deviation from the full SM predictions becomes sizeable very rapidly and it is therefore fair to say that the NLO corrections in the effective theory turn into a sub-leading effect, already at $p_{T} \sim 400 \mathrm{GeV}$. Similar observations have already been made before [22, 23, 24], it is however interesting to see that the differential ratios associated with $p_{T, H}$ and $p_{T, j_{1}}$ are strikingly similar in their characteristics even beyond the one-jet case. In addition, they are also very similar among the different jet bins, suggesting that the relative $1 / p_{T}^{2}$ scaling between the effective and full theory at $\mathrm{LO}[25,26]$ can be 
applied in a more universal manner. In fact if we concentrate on the $p_{T, H}$ predictions, we observe that the suggested scaling for the cross section ratio $R_{m_{t, b}}\left(p_{T, H}\right) \equiv R_{m_{t, b}}\left(p_{T, H}^{2}\right)$ holds to a fairly good extent. For example, at $p_{T, H} \approx 400 \mathrm{GeV}$, the mass effects reduce the cross section to roughly $60 \%$ of the effective theory result. At $p_{T, H}>1 \mathrm{TeV}$, this reduction then turns into an one-order of magnitude effect, which fixes the related ratio at a value of

$$
\frac{R_{m_{t, b}}\left(p_{T, H}=1.0 \mathrm{TeV}\right)}{R_{m_{t, b}}\left(p_{T, H}=0.4 \mathrm{TeV}\right)} \approx \frac{10 \%}{60 \%}=\frac{1}{6}
$$

The above number (as given by our computation) can be compared with the number one expects from exploiting the relative scaling property between the effective and full theory predictions. Based on the additional suppression of the full result by two powers of $p_{T, H}$, the expected value for the same cross section ratio amounts to $(400 \mathrm{GeV} / 1000 \mathrm{GeV})^{2}=4 / 25$, which is very close to the value extracted from the theory data. This result for the scaling does not change much among the different jet bins [1].

The impact of finite mass effects can therefore be very dramatic when studying Higgs boson production in gluon-gluon fusion at transverse momenta above $p_{T, H} \approx 200 \mathrm{GeV}$. As the LHC collects more and more statistics allowing for Higgs measurements also at large $p_{T}$, the inclusion of mass effects becomes more and more relevant in particular for boosted Higgs studies [27]. Many more observables and results, also including bottom mass effects at the differential level and the impact of vector boson fusion selection cuts can be found in [1].

\section{References}

[1] N. Greiner, S. Höche, G. Luisoni, M. Schönherr and J. C. Winter, JHEP 1701 (2017) 091 doi:10.1007/JHEP01(2017)091 [arXiv:1608.01195].

[2] G. Cullen et al., Phys. Rev. Lett. 111 (2013) no.13, 131801 doi:10.1103/PhysRevLett.111.131801 [arXiv:1307.4737].

[3] N. Greiner, S. Höche, G. Luisoni, M. Schönherr, J. C. Winter and V. Yundin, JHEP 1601 (2016) 169 doi:10.1007/JHEP01(2016)169 [arXiv:1506.01016].

[4] G. Cullen, N. Greiner, G. Heinrich, G. Luisoni, P. Mastrolia, G. Ossola, T. Reiter and F. Tramontano, Eur. Phys. J. C 72 (2012) 1889 doi:10.1140/epjc/s10052-012-1889-1 [arXiv:1111.2034]; G. Cullen et al., Eur. Phys. J. C 74 (2014) no.8, 3001 doi:10.1140/epjc/s10052-014-3001-5 [arXiv:1404.7096].

[5] P. Nogueira, J. Comput. Phys. 105 (1993) 279. doi:10.1006/jcph.1993.1074

[6] J. Kuipers, T. Ueda, J. A. M. Vermaseren and J. Vollinga, Comput. Phys. Commun. 184 (2013) 1453 doi:10.1016/j.cpc.2012.12.028 [arXiv:1203.6543].

[7] G. Cullen, M. Koch-Janusz and T. Reiter, Comput. Phys. Commun. 182 (2011) 2368 [arXiv:1008.0803].

[8] T. Peraro, Comput. Phys. Commun. 185 (2014) 2771 [arXiv:1403.1229].

[9] P. Mastrolia, E. Mirabella and T. Peraro, JHEP 1206 (2012) 095 Erratum: [JHEP 1211 (2012) 128] doi:10.1007/JHEP11(2012)128, 10.1007/JHEP06(2012)095 [arXiv:1203.0291]; H. van Deurzen, G. Luisoni, P. Mastrolia, E. Mirabella, G. Ossola and T. Peraro, JHEP 1403 (2014) 115 doi:10.1007/JHEP03(2014)115 [arXiv:1312.6678]; 
[10] P. Mastrolia, G. Ossola, T. Reiter and F. Tramontano, JHEP 1008 (2010) 080 doi:10.1007/JHEP08(2010)080 [arXiv:1006.0710].

[11] G. Ossola, C. G. Papadopoulos and R. Pittau, Nucl. Phys. B 763 (2007) 147 [hep-ph/0609007]. G. Ossola, C. G. Papadopoulos and R. Pittau, JHEP 0805 (2008) 004 [arXiv:0802.1876]. P. Mastrolia, G. Ossola, C. G. Papadopoulos and R. Pittau, JHEP 0806 (2008) 030 [arXiv:0803.3964].

[12] T. Binoth, J.-P. Guillet, G. Heinrich, E. Pilon and T. Reiter, Comput. Phys. Commun. 180 (2009) 2317 doi:10.1016/j.cpc.2009.06.024 [arXiv:0810.0992]; G. Cullen, J. P. Guillet, G. Heinrich, T. Kleinschmidt, E. Pilon, T. Reiter and M. Rodgers, Comput. Phys. Commun. 182 (2011) 2276 doi:10.1016/j.cpc.2011.05.015 [arXiv:1101.5595]; J. P. Guillet, G. Heinrich and J. F. von Soden-Fraunhofen, Comput. Phys. Commun. 185 (2014) 1828 doi:10.1016/j.cpc.2014.03.009 [arXiv:1312.3887].

[13] G. Heinrich, G. Ossola, T. Reiter and F. Tramontano, JHEP 1010 (2010) 105 doi:10.1007/JHEP10(2010)105 [arXiv:1008.2441].

[14] G. J. van Oldenborgh, Comput. Phys. Commun. 66 (1991) 1. doi:10.1016/0010-4655(91)90002-3; R. K. Ellis and G. Zanderighi, JHEP 0802 (2008) 002 doi:10.1088/1126-6708/2008/02/002 [arXiv:0712.1851]; S. Carrazza, R. K. Ellis and G. Zanderighi, Comput. Phys. Commun. 209 (2016) 134 doi:10.1016/j.cpc.2016.07.033 [arXiv:1605.03181].

[15] A. van Hameren, Comput. Phys. Commun. 182 (2011) 2427 doi:10.1016/j.cpc.2011.06.011 [arXiv:1007.4716].

[16] T. Gleisberg, S. Hoeche, F. Krauss, M. Schonherr, S. Schumann, F. Siegert and J. Winter, JHEP 0902 (2009) 007 doi:10.1088/1126-6708/2009/02/007 [arXiv:0811.4622].

[17] T. Gleisberg and S. Hoeche, JHEP 0812 (2008) 039 doi:10.1088/1126-6708/2008/12/039 [arXiv:0808.3674].

[18] T. Binoth et al., Comput. Phys. Commun. 181 (2010) 1612 doi:10.1016/j.cpc.2010.05.016 [arXiv:1001.1307]. S. Alioli et al., Comput. Phys. Commun. 185 (2014) 560 doi:10.1016/j.cpc.2013.10.020 [arXiv:1308.3462].

[19] Z. Bern, L. J. Dixon, F. Febres Cordero, S. Höche, H. Ita, D. A. Kosower and D. Maitre, Comput. Phys. Commun. 185 (2014) 1443 doi:10.1016/j.cpc.2014.01.011 [arXiv:1310.7439].

[20] M. Cacciari and G. P. Salam, Phys. Lett. B 641 (2006) 57 doi:10.1016/j.physletb.2006.08.037 [hep-ph/0512210]; M. Cacciari, G. P. Salam and G. Soyez, JHEP 0804 (2008) 063 doi:10.1088/1126-6708/2008/04/063 [arXiv:0802.1189].

[21] M. Cacciari, G. P. Salam and G. Soyez, Eur. Phys. J. C 72 (2012) 1896 doi:10.1140/epjc/s10052-012-1896-2 [arXiv:1111.6097].

[22] M. Buschmann, D. Goncalves, S. Kuttimalai, M. Schonherr, F. Krauss and T. Plehn, JHEP 1502 (2015) 038 doi:10.1007/JHEP02(2015)038 [arXiv:1410.5806].

[23] R. Frederix, S. Frixione, E. Vryonidou and M. Wiesemann, JHEP 1608 (2016) 006 doi:10.1007/JHEP08(2016)006 [arXiv:1604.03017].

[24] J. R. Andersen et al., [arXiv:1605.04692].

[25] S. Forte and C. Muselli, JHEP 1603 (2016) 122 doi:10.1007/JHEP03(2016)122 [arXiv:1511.05561].

[26] F. Caola, S. Forte, S. Marzani, C. Muselli and G. Vita, JHEP 1608 (2016) 150 doi:10.1007/JHEP08(2016)150 [arXiv:1606.04100].

[27] CMS Collaboration, CMS-PAS-HIG-17-010. 